УДК 519.21

\title{
Study of a Logarithmic Barrier Approach for Linear Semidefinite Programming
}

\author{
Assma Leulmi* \\ Department of Mathematics \\ University of Skikda \\ Algeria \\ Bachir Merikhi \\ Djamel Benterki \\ Department of Mathematics \\ Ferhat Abbas Setif University \\ Algeria
}

Received 16.04.2017, received in revised form 06.12.2017, accepted 07.03.2018

\begin{abstract}
In this paper, we present a logarithmic barrier interior-point method for solving a semidefinite programming problem. Newton's method is used to compute the descent direction, and minorant function are used as an efficient alternative to line search methods to determine the displacement step along the direction in order to reduce the computation cost.
\end{abstract}

Keywords: semidefinite programming, interior-point methods, logarithmic barrier methods, line search. DOI: 10.17516/1997-1397-2018-11-3-300-312.

\section{Background information}

In semidefinite programming (SDP) we minimize a linear function subject to the constraint that an affine combination of symmetric matrices is positive semidefinite. Such a constraint is nonlinear and nonsmooth, but convex, so positive definite programs are convex optimization problems. Semidefinite programming unifies several standard problems (eg, linear and quadratic programming) and finds many applications in engineering. Although semidefinite programs are much more general than linear programs, they are just as easy to solve. Most interior-point methods for linear programming have been generalized to semidefinite programs. As in linear programming, these methods have polynomial worst-case complexity, and perform very well in practice.

Interior-point methods [1] are one of the efficient methods developed to solve linear, semidefinite and nonlinear programming problems.

In this paper, we particularly propose a logarithmic barrier interior-point method for solving semidefinite programming problem (SDP). In fact, the main difficulty to be anticipated in establishing an iteration in such a method will come from the determination and computation of the displacement step. Various approaches are developed to overcome this difficulty. It is known $[4,5]$ that the computation of the displacement step is expensive specifically while using line search methods.

The purpose of this paper is to propose alternative ways to determine the displacement step which is more efficient than classical line-searches.

*as_smaleulmi@yahoo.fr

(c) Sibe-rian Federal University. All rights reserved 
The SDP problem and its dual are defined as

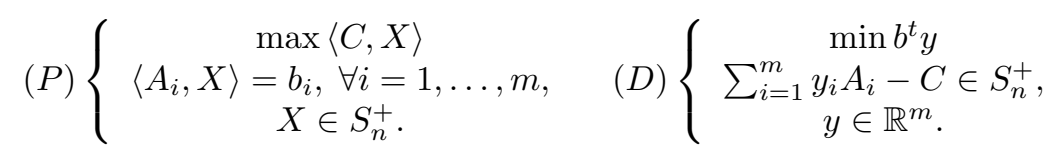

Where $S_{n}^{+}$designs the cone of the symmetrical semidefinite positive $n \times n$ matrix, matrices $C, A_{i}$, with $i=1, \ldots, m$, are the given symmetrical matrices and $b \in \mathbb{R}^{m}$.

And we denote by $\langle C, X\rangle$, the trace of the matrix $\left(C^{t} X\right)$. It is recalled that $\langle.,$.$\rangle corresponds$ to an inner product on the space of $n \times n$ matrices.

Now, we make assumptions about the primal-dual pair $(P, D)$. First, we define the following feasibility sets

$$
\begin{aligned}
& Y=\left\{y \in \mathbb{R}^{m}: \sum_{i=1}^{m} y_{i} A_{i}-C \in S_{n}^{+}\right\}, \\
& \widehat{Y}=\left\{y \in \mathbb{R}^{m}: \sum_{i=1}^{m} y_{i} A_{i}-C \in \operatorname{int}\left(S_{n}^{+}\right)\right\}, \\
& F=\left\{X \in S_{n}^{+}:\left\langle A_{i}, X\right\rangle=b_{i}, \forall i=1, \ldots, m\right\}, \\
& \widehat{F}=\left\{X \in F: X \in \operatorname{int}\left(S_{n}^{+}\right)\right\} .
\end{aligned}
$$

With $\operatorname{int}\left(S_{n}^{+}\right)$is the set of the symmetrical definite positive $n \times n$ matrices.

Assumption 1.1. The system of equations $\left\langle A_{i}, Y\right\rangle=b_{i}, i=1, \ldots, m$ is of rank $m$.

Assumption 1.2. The sets $\widehat{Y}$ and $\widehat{F}$ are not empty.

Let $r>0$ be a barrier parameter and $\left.\left.f_{r}: \mathbb{R}^{m} \rightarrow\right]-\infty,+\infty\right]$ be a barrier function defined as

$$
f_{r}(y)= \begin{cases}b^{t} y-r \ln \left[\operatorname{det}\left(\sum_{i=1}^{m} y_{i} A_{i}-C\right)\right]+n r \ln r & \text { if } y \in \widehat{Y} \\ +\infty & \text { if not. }\end{cases}
$$

Then solving problem $(D)$ is equivalent to solving the perturbed unconstrained optimization problems

$$
\left(D_{r}\right)\left\{\begin{aligned}
\min & f_{r}(y) \\
y & \in \mathbb{R}^{m}
\end{aligned}\right.
$$

The focus of this paper is on solving the perturbed problem (1). This paper is organized as follows. In Section 2, we briefly recall some results in linear semidefinite programming and give some preliminary results. In Section 3, after considering the existence and uniqueness of the optimal solution of perturbed problem (1), we show the convergence of the last problem to problem $(D)$ in the sense that the optimal solution of problem (1) approaches the optimal solution of $(D)$ as $r \rightarrow 0$. The solution of this problem is of descent type, defined by $y_{k+1}=y_{k}+t_{k} d_{k}$ where $d_{k}$ is the descent direction and $t_{k}$ is the displacement step.

In Section 4, we propose an interior-point algorithm for solving the perturbed problem (1). Newton's method is applied to compute the descent direction $d$ by solving the linear system resulted from the optimality conditions associated with problem (1). As an effective and less expensive alternative to line search methods, the so-called minorants function are used to determine the displacement step $t$ along the descent direction. Section 5 contains some concluding remarks. 


\section{Background and preliminary results}

This section provides the necessary background for the upcoming development. In Subsection 2.1, we review some results in linear semidefinite programming. We refer the reader to [9,12], for more details. In Subsection 2.2, we review some statistical inequalities.

\subsection{A brief background in linear semidefinite programming}

We know that (see $[1,10])$

a) the sets of the $(P)$ and $(D)$ optimal solutions are non empty convex, compact sets;

b) if $\bar{X}$ is an optimal solution of $(P)$, then $\bar{y}$ is an optimal solution of $(D)$ if and only if $\bar{y} \in Y$ and $\left(\sum_{i=1}^{m} \bar{y}_{i} A_{i}-C\right) \bar{X}=0$;

c) if $\bar{y}$ is an optimal solution of $(D)$, then $\bar{X}$ is an optimal solution of $(P)$ if and only if $\bar{X} \in F$ and $\left(\sum_{i=1}^{m} \bar{y}_{i} A_{i}-C\right) \bar{X}=0$.

In these conditions, the $(D)$ problem resolution permits to obtain that of $(P)$ and vice versa.

\subsection{Preliminary inequalities}

Let $x_{1}, x_{2}, \ldots, x_{n} \in \mathbb{R}$ be a sample of size $n$, then its mean $\bar{x}$ and its standard deviation $\sigma_{x}$ are respectively defined as

$$
\bar{x}=\frac{1}{n} \sum_{i=1}^{n} x_{i} \text { and } \sigma_{x}^{2}=\frac{1}{n} \sum_{i=1}^{n} x_{i}^{2}-\bar{x}^{2}=\frac{1}{n} \sum_{i=1}^{n}\left(x_{i}-\bar{x}\right)^{2} .
$$

Proposition 1. Assume that $x \in \mathbb{R}^{n}$, then we have

$$
\bar{x}-\sigma_{x} \sqrt{n-1} \leqslant \min _{1 \leqslant k \leqslant n} x_{k} \leqslant \bar{x}-\frac{\sigma_{x}}{\sqrt{n-1}} \text { and } \bar{x}+\frac{\sigma_{x}}{\sqrt{n-1}} \leqslant \max _{1 \leqslant k \leqslant n} x_{k} \leqslant \bar{x}+\sigma_{x} \sqrt{n-1} .
$$

In particular, if $x_{k}>0$ for all $k=1, \ldots, n$, then we also have

$$
n \ln \left(\bar{x}-\sigma_{x} \sqrt{n-1}\right) \leqslant A \leqslant \sum_{i=1}^{n} \ln \left(x_{i}\right) \leqslant B \leqslant n \ln (\bar{x}),
$$

with

$$
\begin{aligned}
& A=(n-1) \ln \left(\bar{x}+\frac{\sigma_{x}}{\sqrt{n-1}}\right)+\ln \left(\bar{x}-\sigma_{x} \sqrt{n-1}\right), \\
& B=\ln \left(\bar{x}+\sigma_{x} \sqrt{n-1}\right)+(n-1) \ln \left(\bar{x}-\frac{\sigma_{x}}{\sqrt{n-1}}\right) .
\end{aligned}
$$

The first statement in Proposition 1 is due to [12] and the second statement is due to [9].

\section{The theoretical aspects of perturbed problem}

In this section, we show that perturbed problem (1) has at least one optimal solution and that this optimal solution converges to the optimal solution of problem $(D)$ when $r$ goes to 0 .

Firstly, we start with the fundamental properties of $f_{r}$. For $y \in \widehat{Y}$, let's introduce the symmetrical definite positive matrix $B(y)$ of rank $m$ and the lower triangular matrix $L(y)$ such 
that $B(y)=\sum_{i=1}^{m} y_{i} A_{i}-C=L(y) L^{t}(y)$, and let's define, for $i, j=1, \ldots, m$

$$
\begin{aligned}
& \widehat{A}_{i}(y)=[L(y)]^{-1} A_{i}\left[L^{t}(y)\right]^{-1} \\
& b_{i}(y)=\operatorname{trace}\left(\widehat{A}_{i}(y)\right)=\operatorname{trace}\left(A_{i} B^{-1}(y)\right) \\
& \Delta_{i j}(y)=\operatorname{trace}\left(B^{-1}(y) A_{i} B^{-1}(y) A_{j}\right)=\operatorname{trace}\left(\widehat{A}_{i}(y) \widehat{A}_{j}(y)\right) .
\end{aligned}
$$

Thus, $b(y)=\left(b_{i}(y)\right)_{i=1, \ldots, m}$ is a vector of $\mathbb{R}^{m}$ and $\Delta(y)=\left(\Delta_{i j}(y)\right)_{i, j=1, \ldots, m}$ is a symmetrical matrix of rank $m$.

The previous notation will be used in the expressions of the gradient and the Hessian $H$ of $f_{r}$. To show that perturbed problem (1) has a solution, it is sufficient to show that $f_{r}$ is inf-compact.

Theorem 2 ( [6]). The function $f_{r}$ is twice continuously differentiable on $\widehat{Y}$. Actually, for all $y \in \widehat{Y}$ we have

(a) $\nabla f_{r}(y)=b-r b(y)$.

(b) $H=\nabla^{2} f_{r}(y)=r \Delta(y)$.

(c) The matrix $\Delta(y)$ is definite positive.

Since $f_{r}$ is strictly convex, (1) has at most one optimal solution, then, we give the following definition.

Definition 1. Let $h$ be a function defined from $\mathbb{R}^{m}$ to $\mathbb{R} \cup\{\infty\}$ and $\alpha \geqslant 0$. Then

(i) The set $C_{\alpha}(h)=\left\{y \in \mathbb{R}^{m}: h(y) \leqslant \alpha\right\}$ is called the $\alpha$-level set of $h$.

(ii) The function $h$ is called inf-compact if the level sets $C_{\alpha}(h)$ are compact for all $\alpha>0$.

(iii) The recession function of $h$ is the function $(h)_{\infty}: \mathbb{R}^{m} \rightarrow \mathbb{R} \cup\{\infty\}$ defined by

$$
(h)_{\infty}(\triangle y)=\lim _{t \rightarrow+\infty}\left[\frac{f_{r}(y+t \triangle y)-f_{r}(y)}{t}\right] .
$$

(iv) The recession cone of $h$ is the 0 -level set of the recession function of $h$, denoted by $C_{0}\left((h)_{\infty}\right)$.

As the function $f_{r}$ takes the value $+\infty$ on the boundary of $Y$ and is differentiable on $\widehat{Y}$, then it is lower semi-continuous. In order to prove that (1) has one optimal solution, it suffices to prove that recession cone of $f_{r}$ defined by

$$
C_{0}\left(\left(f_{r}\right)_{\infty}\right)=\left\{d \in \mathbb{R}^{m},\left(f_{r}\right)_{\infty}(d) \leqslant 0\right\}
$$

is reduced to zero i.e.,

$$
d=0 \text { if }\left(f_{r}\right)_{\infty}(d) \leqslant 0
$$

where $\left(f_{r}\right)_{\infty}$ is defined for $y \in \widehat{Y}$ as

$$
\left(f_{r}\right)_{\infty}(d)=\lim _{t \rightarrow+\infty}\left[\xi(t)=\frac{f_{r}(y+t d)-f_{r}(y)}{t}\right] .
$$

This needs to the following proposition.

Proposition 3 ( [6]). If $b^{T} d \leqslant 0$ and $\sum_{i=1}^{m} d_{i} A_{i} \in \widehat{Y}$ then $d=0$, where $d$ is the descent direction.

As $f_{r}$ is inf-compact and strictly convex, therefore the approximated problem admits a unique optimal solution.

We denote by $y(r)$ or $y_{r}$ the unique optimal solution of perturbed problem (1). 


\subsection{Convergence of perturbed problem to (D)}

Now, we show that perturbed problem (1) converges to problem $(D)$ as $r \rightarrow 0$. We have the following lemma.

Lemma 1. For $r>0$, let perturbed problem (1) have $y(r)$ as an optimal solution, then problem (D) has $y^{*}=\lim _{r \rightarrow 0} y(r)$ as an optimal solution.

Proof. Let $y \in \widehat{Y}$ be arbitrary and $r>0$ be given. Let us introduce the function $f: \mathbb{R}^{m} \times \mathbb{R} \rightarrow$ ]$-\infty,+\infty]$, defined by

$$
\psi(y, r)=f_{r}(y)=\left\{\begin{array}{lll}
f_{r}(y) & \text { if } & r>0 \\
b^{t} y & \text { if } & r=0, y \in Y \\
+\infty & \text { if not. } &
\end{array}\right.
$$

It is easy to verify that the function $f$ is convex and lower semi-continuous on $\mathbb{R}^{m} \times \mathbb{R}$, see [11], then there exists an optimal solution $y_{r}$ of (1) such that

$$
\nabla_{y} f_{r}\left(\bar{y}_{r}\right)=\nabla_{y} f\left(\bar{y}_{r}, r\right)=0
$$

since $f(y)=f(y, 0)$, we have

$f(y) \geqslant \psi\left(\bar{y}_{r}, r\right)+\left(y-\bar{y}_{r}\right)^{T} \nabla_{y} \psi\left(\bar{y}_{r}, r\right)+(0-\mu) \frac{\partial}{\partial_{r}} \psi\left(\bar{y}_{r}, r\right) \geqslant \psi\left(\bar{y}_{r}, r\right)-\mu \frac{\partial}{\partial_{r}} \psi\left(\bar{y}_{r}, r\right) \geqslant f\left(\bar{y}_{r}\right)-r n$.

which implies

$$
f\left(\bar{y}_{r}\right)-r n \leqslant \min _{y \in \widehat{Y}} f(y) \leqslant f\left(\bar{y}_{r}\right)
$$

On the other hand, we have

$$
f\left(\bar{y}_{r}\right) \geqslant \min _{y \in \widehat{Y}} f(y)
$$

When $r$ tends to 0 , we conclude that

$$
f\left(\bar{y}_{r}\right)=\min _{y \in \widehat{Y}} f(y)
$$

Therefore $\bar{y}$ is an optimal solution of $(D)$.

Remark 1. We know that if one of the problems $(D)$ and $(P)$ has an optimal solution, and the values of their objective functions are equal and finite, the other problem has an optimal solution.

\section{The numerical aspects of perturbed problem}

\subsection{Newton descent direction}

In this part, we are interested in the numerical solution of the problem (1). With the presence of the barrier function, the problem (1) can be considered as without constraints. So, interior point methods of types logarithmic barrier are conceived for solving this problem type while being based on the optimality conditions which are necessary and sufficient. $y_{r}$ is an optimal solution of (1) if it satisfies the following condition

$$
\nabla f_{r}\left(y_{r}\right)=0 .
$$


To solve Eq. 3, we use the Newton's approach which means to find at each iteration a vector $y_{r k}+d_{k}$ checking the following linear system

$$
\left[\nabla^{2} f_{r}\left(y_{r}\right)\right] d=-\nabla f_{r}\left(y_{r}\right)
$$

By virtue of the Theorem 2, the linear system is equivalent to the system

$$
\Delta\left(y_{r}\right) d=b\left(y_{r}\right)-\frac{1}{r} b
$$

where $b(y)$ and $\Delta(y)$ are defined in $(3)$.

As $H_{k}=\nabla^{2} f_{r}\left(y_{r}\right)$ is a symmetric positive definite matrix, the Cholesky methods and the conjugate gradient methods are the best convenient for solving the system (4).

To ensure the convergence of the algorithm to wards an optimal solution $y^{*}$ of (1), it should be made sure that all the iterate $y_{r k}+d_{k}$ remains strictly feasible. For that, we introduce a displacement step $t_{k}$ checking the condition

$$
B(y)=\sum_{i=1}^{m}\left(y_{r k}+t_{k} d_{k}\right) A_{i}-C>0 .
$$

A prototype algorithm for solving problem (1) is formally stated in the next Algorithm. For the sake of simplicity we drop the index $r$ from $y_{r}$ and $y_{r k}$, and write y instead of $y_{r}$ and $y_{k}$ instead of $y_{r k}$.

\subsection{Prototype algorithm for solving the perturbed problem}

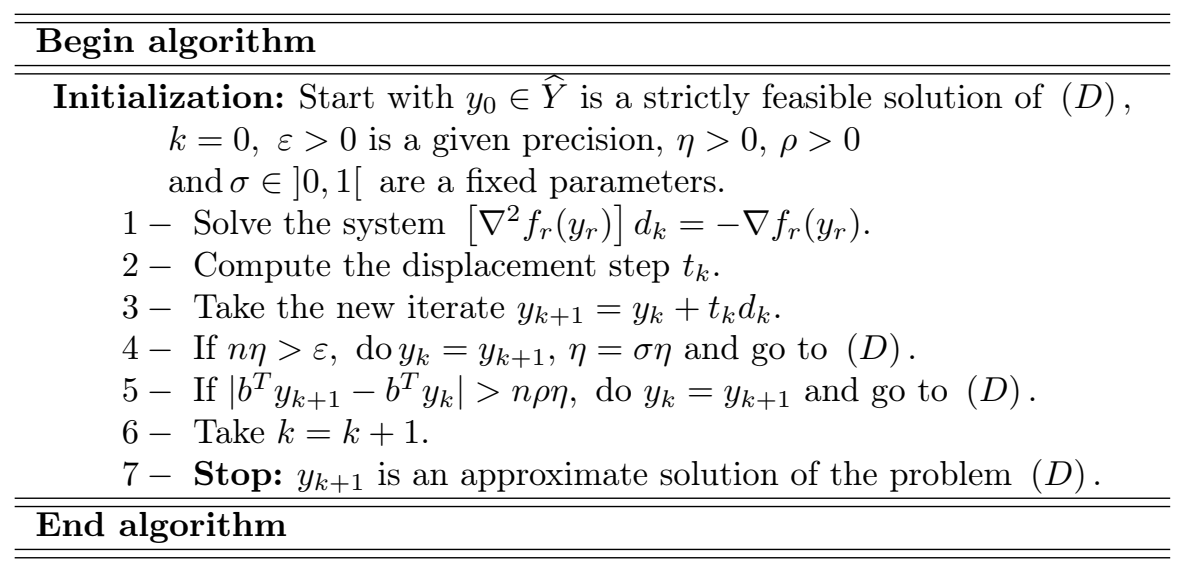

We know from the preceding facts, that the optimal solution of problem (1) is an approximation of the solution of $(D)$. More $r$ tends to zero more approximation is good. Unfortunately, when $r$ approaches zero, the problem (1) becomes ill-conditioned. It is the reason for which we utilize at the beginning of the iteration the values of $r$ that are not near to zero, that verifies $n r<\varepsilon$. We can explain interpretation the update $r$ as follows : if $y(r)$ is an exact solution of (1), it is non necessary to keep on the calculus of the iterates when $\left|b^{t} y-b^{t} \bar{y}\right| \leqslant \rho n r$.

\subsection{Computation of the displacement step}

The most known methods used to compute the optimal displacement step $t_{k}$ are the line search methods, which require minimizing the unidimensional function

$$
\phi(t)=\min f_{r}(y+t d) .
$$


The most used methods of the type line search are those of Goldstein-Armijo, Fibonacci, etc. Unfortunately, these methods are expensive in computational volume, and even inapplicable to semidefinite problems.

To avoid this difficulty, we exploit the idea suggested by J. P. Crouzeix and B. Merikhi [6] which approaches the function $\theta(t)$ defined as

$$
\theta(t)=\frac{1}{r}\left[f_{r}(y+t d)-f_{r}(y)\right], y+t d \in \widehat{Y},
$$

by the simple minorant function giving at each iteration $k$, a displacement step $t_{k}$ in an easy way, simple and much less expensive than line search methods.

To simplify the notations, we consider

$$
B=B(y)=\sum_{i=1}^{m} y_{i} A_{i}-C \text { and } H=\sum_{i=1}^{m} d_{i} A_{i}
$$

where $B$ is a symmetrical and positive definite, there exists a lower triangular matrix $L$ such that $B=L L^{T}$.

Next, let's put $E=L^{-1} H\left(L^{-1}\right)^{T}$, since $d \neq 0$, the assumption 1 implies that $H \neq 0$ and then $E \neq 0$, with this notation, for any $t>0, I+t E$ is positive definite. Let's denote by $\lambda_{i}$ the eigenvalues of the symmetric matrix $E$.

Remark 2. It is necessary that the point $y+t d$ still in $\widehat{Y}$ for all $\widehat{Y}$ to keep function $\theta(t)$ well defined. This in turns requires finding $\widehat{t}>0$ such that $y+t d \in \widehat{Y}$ for any $t \in[0, \widehat{t}[$.

Lemma 2. Let $\widehat{t}=\sup \left\{t: 1+t \lambda_{i}>0, i=1, \ldots, n\right\}$. For all $t \in[0, \widehat{t}[$, the following function $\theta(t)$ is well defined

$$
\theta(t)=\sum_{i=1}^{n}\left[t\left(\lambda_{i}-\lambda_{i}^{2}\right)-\ln \left(1+t \lambda_{i}\right)\right], t \in[0, \widehat{t}[
$$

Proof. We have

$$
\theta(t)=\frac{1}{r}\left[f_{r}(y+t d)-f_{r}(y)\right]=\frac{1}{r} t b^{t} d-\ln \operatorname{det}\left(\sum_{i=1}^{m}\left(y_{i}+t d\right) A_{i}-C\right)+\ln \operatorname{det}\left(\sum_{i=1}^{m} y_{i} A_{i}-C\right)
$$

or

$$
\sum_{i=1}^{m}\left(y_{i}+t d\right) A_{i}-C=\left(\sum_{i=1}^{m} y_{i} A_{i}-C\right)+t \sum_{i=1}^{m} d_{i} A_{i}=B+t H=B\left(I+t B^{-1} H\right) .
$$

Then

$$
\begin{aligned}
\theta(t) & =\frac{t}{r} b^{t} d-\ln \operatorname{det} B(y)-\ln \operatorname{det}\left(I+t B^{-1} H\right)+\ln \operatorname{det}(B(y)), \\
\theta(t) & =\frac{t}{r} b^{t} d-\ln \operatorname{det}\left(I+t B(y)^{-1} H\right)=\frac{t}{r} b^{t} d-\ln \operatorname{det}(I+t E),
\end{aligned}
$$

but $B=L L^{t}$, then $B^{-1}=\left(L^{t}\right)^{-1} L^{-1}$.

Since $\nabla f_{r}(y)=b-r b(y)$, we have $b_{i}(y)=\operatorname{trace}\left(\widehat{A}_{i}(y)\right)=\operatorname{trace}\left(A_{i} B^{-1}(y)\right)$ and $\left[\nabla^{2} f_{r}\left(y_{r}\right)\right] d=$ $=-\nabla f_{r}\left(y_{r}\right)$.

$$
d^{t} b=d^{t} \nabla f_{r}(y)+r d^{t} b(y),
$$

Due to the fact that the direction $d$ satisfies $\left[\nabla^{2} f_{r}\left(y_{r}\right)\right] d=-\nabla f_{r}\left(y_{r}\right)$, then, we have

$$
d^{t}\left[\nabla^{2} f_{r}\left(y_{r}\right)\right] d=-d^{t} \nabla f_{r}\left(y_{r}\right)
$$


Substituting (9) into (8), we get

$$
\begin{aligned}
d^{t} b & =d^{t} \nabla f_{r}(y)+r d^{t} b(y)=-d^{t}\left[\nabla^{2} f_{r}\left(y_{r}\right)\right] d+r d^{t} b(y)= \\
& =-d^{t}\left[\nabla^{2} f_{r}\left(y_{r}\right)\right] d+r \sum d^{t} \operatorname{trace}\left(A_{i} B^{-1}\left(y_{r}\right)\right)=-d^{t}\left[\nabla^{2} f_{r}\left(y_{r}\right)\right] d+r \operatorname{trace}(E),
\end{aligned}
$$

but we have

$$
\begin{aligned}
d^{t}\left[\nabla^{2} f_{r}\left(y_{r}\right)\right] d & =d^{t}\left[r \Delta\left(y_{r}\right)\right] d=r d^{t}\left[\operatorname{trace}\left(B^{-1}\left(y_{r}\right) A_{i} B^{-1}\left(y_{r}\right) A_{j}\right)\right] d= \\
& =r \sum_{i=1}^{n} d_{i}^{2} \operatorname{trace}\left(B^{-1}\left(y_{r}\right) A_{i} B^{-1}\left(y_{r}\right) A_{j}\right)=r \operatorname{trace}\left(E^{2}\right)
\end{aligned}
$$

Then, by substituting in (10), we get

$$
d^{t} b=r \operatorname{trace}(E)-r \operatorname{trace}\left(E^{2}\right) .
$$

Substituting (11) into (7), we obtain

$$
\theta(t)=t\left(\operatorname{trace}(E)-\operatorname{trace}\left(E^{2}\right)\right)-\ln \operatorname{det}(I+t E) .
$$

Let's designate by $\lambda_{i}$, the characteristic values of the symmetrical matrix $E$, so

$$
\theta(t)=\sum_{i=1}^{n}\left[t\left(\lambda_{i}-\lambda_{i}^{2}\right)-\ln \left(1+t \lambda_{i}\right)\right], t \in[0, \widehat{t}[,
$$

with $\widehat{t}=\sup \left[t: 1+t \lambda_{i}>0\right.$ for all $\left.i\right]$. The proof is complete.

A lower bound $\widehat{t}_{0}$ of $\widehat{t}$ is based on the first statement of Proposition 1 and is given by

$$
\widehat{t}_{0}=\sup \left[t: 1+t \alpha_{0}>0\right], \text { with } \alpha_{0}=\bar{\lambda}-\frac{\sigma_{\lambda}}{\sqrt{n-1}},
$$

where, as defined in Section $2, \bar{\lambda}=\frac{1}{n} \sum_{i=1}^{n} \lambda_{i}$ and $\sigma_{\lambda}^{2}=\frac{1}{n} \sum_{i=1}^{n} \lambda_{i}^{2}-\bar{\lambda}^{2}$.

Another bound $\widehat{t}_{2}$ is based on the inequality $\left|\lambda_{i}\right| \leqslant\|\lambda\|$ for $i=1, \ldots, n$, and is given by

$$
\widehat{t_{2}}=\sup \left[t: 1+t \alpha_{2}>0\right] \text {, with } \alpha_{2}=\|\lambda\| \text {. }
$$

Unfortunately, it does not exist an explicit formula that gives $t_{\text {opt }}$, and the resolution of the equation $\theta^{\prime}\left(t_{\text {opt }}\right)=0$ through iterative methods need at each iteration to calculate $\theta$ and $\theta^{\prime}$. These calculations are too expensive because the expression of $\theta$ in (12) contains the determinant that is difficult to calculate and (6) necessitates the knowledge of the characteristic values of $E$. It is a numerical problem of large size. These difficult conduct us to look for other new alternatives. From the data of the matrix $E$, it is easy to obtain the following quantities

The computation of the displacement step by classical line-search methods is undesirable and in general impossible.

Now, we look for a minorant function $G$ of the function $\theta$ on $\left[0, \widehat{t}_{0}\right.$ [ which can be used as an lower approximation of $\theta$. Such an lower approximation may be more efficient to manipulate than $\theta$. The function $\widetilde{\theta}$ is chosen to be simple and close enough to $\theta$ and to satisfy the following properties

$$
G(0)=0 \text { and } G^{\prime \prime}(0)=-G^{\prime}(0)=\|\lambda\|^{2},
$$

where $G^{\prime}$ and $G^{\prime \prime}$ denote the first and the second derivative of $G$ respectively.

Based on Proposition 1, we give in the following, new notions of the non expensive minorant function for $\theta$, that offers displacement steps with a simple technique. 


\section{The first minorant function}

This strategy consists to minimize the minorant approximations $G$ of $\theta$ over $[0, \hat{t}[$. To be efficient, this minorant approximation needs to be simple and sufficiently near $\theta$. In our case, it requires

$$
0=\theta(0),\|\lambda\|^{2}=\theta^{\prime \prime}(0)=-\theta^{\prime}(0)
$$

We may define a minorant function $G_{0}$ on $[0, \widehat{t}[$ by

$$
G_{0}(t)=\gamma_{0} t-\ln \left(1+\beta_{0} t\right)-(n-1) \ln \left(1+\alpha_{0} t\right),
$$

with $\gamma_{0}=n \bar{\lambda}-\|\lambda\|^{2}, \alpha_{0}=\bar{\lambda}-\frac{\sigma_{\lambda}}{\sqrt{n-1}}$ and $\beta_{0}=\bar{\lambda}+\sigma_{\lambda} \sqrt{n-1}$.

The logarithms are well defined when $t \leqslant \widehat{t}_{0}$ with $\widehat{t}_{0}=\left\{\begin{array}{cl}-\frac{1}{\alpha_{0}} & \text { if } \alpha_{0}<0, \\ +\infty & \text { if not. }\end{array}\right.$

Theorem 4. We have $G_{0}(t) \leqslant \theta(t), \forall t \in[0, \widehat{t}[$.

Proof. Let $x_{1}, x_{2}>0$. Using the second statement of Proposition 1, we have

$$
\sum_{i=1}^{n} \ln \left(x_{i}\right) \leqslant \ln \left(\bar{x}+\sigma_{x} \sqrt{n-1}\right)+(n-1) \ln \left(\bar{x}-\frac{\sigma_{x}}{\sqrt{n-1}}\right) .
$$

This implies that

$$
\sum_{i=1}^{n} \ln \left(x_{i}\right)+t\|\lambda\|^{2} \leqslant \ln \left(\bar{x}+\sigma_{x} \sqrt{n-1}\right)+(n-1) \ln \left(\bar{x}-\frac{\sigma_{x}}{\sqrt{n-1}}\right)+t\|\lambda\|^{2},
$$

which in turn implies that

$$
n \bar{\lambda} t-\left(\sum_{i=1}^{n} \ln \left(x_{i}\right)+\|\lambda\|^{2} t\right) \geqslant n \bar{\lambda} t-\left(\ln \left(\bar{x}+\sigma_{x} \sqrt{n-1}\right)+(n-1) \ln \left(\bar{x}-\frac{\sigma_{x}}{\sqrt{n-1}}\right)+\|\lambda\|^{2} t\right) .
$$

Taking $x_{i}=1+t \lambda_{i}$ for any $i=1, \ldots, n$, hence $\bar{x}=1+t \bar{\lambda}$ and $\sigma_{x}=t \sigma_{\lambda}$, we get

$$
\left(n \bar{\lambda}-\|\lambda\|^{2}\right) t-\ln \left(1+\beta_{0} t\right)-(n-1) \ln \left(1+\alpha_{0} t\right) \leqslant\left(n \bar{\lambda}-\|\lambda\|^{2}\right) t-\sum_{i=1}^{n} \ln \left(1+\lambda_{i} t\right),
$$

with $\alpha_{0}=\bar{\lambda}-\frac{\sigma_{\lambda}}{\sqrt{n-1}}$ and $\beta_{0}=\bar{\lambda}+\sigma_{\lambda} \sqrt{n-1}$.

Note that the left-hand side of the above inequality is nothing but the function $\theta(t)$ and the righthand side of the above inequality is nothing but the function $G_{0}(t)$. This means we have shown that $G_{0}(t) \leqslant \theta(t)$ on $[0, \widehat{t}[$.

On the other hand, for any $t \in\left[0, \widehat{t}_{0}[\right.$, we have

$$
\begin{aligned}
\theta(0) & =G_{0}(0)=0, \theta^{\prime}(0)=G_{0}^{\prime}(t)=-\sum_{i=1}^{n} \lambda_{i}^{2} \\
\theta^{\prime \prime}(0) & =G_{0}^{\prime \prime}(t)=\sum_{i=1}^{n} \lambda_{i}^{2}=\operatorname{trace}\left(E^{2}\right)
\end{aligned}
$$


The function $G_{0}$ is strictly convex over $] 0, \widehat{t}_{0}\left[\right.$ and $G_{0}^{\prime}(t)<0$. If $t \rightarrow \infty$ and since $G_{0}$ minimizes $\theta$ which is inf-compact, $G_{0}$ admits a minimum over $\left[0, \widehat{t}_{0}[\right.$.

If $\widehat{t}_{0}<+\infty$ so $G_{0}(t) \rightarrow+\infty$ if $t \rightarrow \widehat{t}_{0}$, Consequently, $G_{0}$ admits a unique minimum over $\left[0, \widehat{t}_{0}\left[\right.\right.$. This minimum is obtained in $t_{\text {opt }}$ such that $G_{0}^{\prime}\left(t_{\text {opt }}\right)=0$.

We are then coming back to solve the second order following equation

$$
t^{2}-2 b t+c t=0
$$

with $b=\frac{1}{2}\left(\frac{n}{\gamma}-\frac{1}{\beta}-\frac{1}{\alpha}\right)$ and $c=-\frac{\|\lambda\|^{2}}{\alpha \beta \gamma}$. Let's take one root of the two roots $t_{\text {opt }}=b \pm \sqrt{b^{2}-c}$ that belong to $[0, \widehat{t}$.

\section{The second minorant function}

We can also think on other more simple functions than $G_{1}$ that does intervene on a unique algorithm. Consider the following functions

$$
G_{1}(t)=\tilde{\gamma} t-\tilde{\delta} \ln (1+\tilde{\alpha} t),
$$

where $\tilde{\alpha}=\alpha_{0}=\bar{\lambda}-\frac{\sigma_{\lambda}}{\sqrt{n-1}}, \tilde{\delta}=\frac{\|\lambda\|^{2}}{\tilde{\alpha}^{2}}$ and $\tilde{\gamma}=\tilde{\delta} \tilde{\alpha}-\|\lambda\|^{2}$. Then

$$
\|\lambda\|^{2}=\tilde{\gamma} \tilde{\alpha}^{2}=\tilde{\delta} \tilde{\alpha}-\tilde{\gamma}
$$

The logarithm is well defined when $t \leqslant \widehat{t}_{0}$ with $\widehat{t}_{0}=\left\{\begin{array}{cc}-\frac{1}{\alpha_{0}} & \text { if } \alpha_{0}<0, \\ +\infty & \text { if not. }\end{array}\right.$

The function $G_{1}$ verifies the following proprieties $G_{1}^{\prime \prime}(0)=-G_{1}^{\prime}(0)=\operatorname{trace}\left(E^{2}\right)$ and $G_{1}(0)=0$, besides $G_{1}(t)<0, \forall t \in\left[0, \widehat{t}_{0}\left[\right.\right.$. Or, since $G_{1}$ is convex and admits a unique minimum over $\left[0, \widehat{t}_{0}\left[\right.\right.$, which can be obtained by resolving the equation $G_{1}^{\prime}(t)=0$, then we obtained

$$
\bar{\alpha}_{1}=\frac{\tilde{\delta}}{\tilde{\gamma}}-\frac{1}{\tilde{\alpha}} .
$$

\section{The third minorat function}

The idea is to use the known inequality following mathematical analysis

$$
\left(\|\lambda\|-\sum_{i=1}^{n} \lambda_{i}\right) t-\ln (1+t\|\lambda\|) \leqslant-\sum_{i=1}^{n} \ln \left(1+t \lambda_{i}\right) .
$$

Replacing in (6), we obtain

$$
\theta(t) \geqslant-\|\lambda\|(\|\lambda\|-1) t-\ln (1+t\|\lambda\|),
$$

then

$$
G_{2}(t)=-\|\lambda\|(\|\lambda\|-1) t-\ln \left(1+\alpha_{2} t\right),
$$

with $\alpha_{2}=\|\lambda\|$ defined at $\left[0, \widehat{t}_{2}\left[\right.\right.$ with $\widehat{t}_{2}=\frac{-1}{\|\lambda\|}$.

Proposition 5. For any $t \in\left[0, \widehat{t}_{2}[\right.$ we have

a. $G_{2}(0)=\theta(0)=0$ and $G_{2}^{\prime}(0)=\theta^{\prime}(0)=-\|\lambda\|^{2}<0$. 
b. $G_{2}^{\prime \prime}(0)=\theta^{\prime \prime}(0)=\|\lambda\|^{2}>0$.

c. $\theta(t) \geqslant G_{2}(t)$.

Proof. 1) (a) and (b) are obvious.

2) For proving $(c)$, we consider the function

$$
h(t)=G_{2}(t)-\theta(t)=\left(\|\lambda\|-\sum_{i=1}^{n} \lambda_{i}\right) t-\ln (1+t\|\lambda\|)+\sum_{i=1}^{n} \ln \left(1+t \lambda_{i}\right) .
$$

We have by definition $h(0)=0$ and to study the sign of the function $h$ we distinguish two cases

1. If there exists $i$ such that $\|\lambda\|=-\lambda_{i}$, then $h(t)=0$ for all $t \in[0, \widehat{t}$.

2. Otherwise, it is known that $-\|\lambda\| \leqslant \lambda_{i} \leqslant\|\lambda\|$. In addition

$$
h^{\prime}(t)=t \sum_{i=1}^{n} \lambda_{i}^{2}\left[\left(1+t \lambda_{i}\right)^{-1}-(1+t\|\lambda\|)^{-1}\right] \text {. }
$$

Since $1+t \lambda_{i} \leqslant 1+t\|\lambda\|$ for all $i$, then $h^{\prime}(t)<0$ for all $t \in[0, \widehat{t}[$. Hence the function $h(t)$ is strictly increasing and $h(0)=0$. Then $h(t) \leqslant 0$ for all $t \in[0, \widehat{t}$, which gives

$$
\theta(t) \geqslant G_{2}(t) \text { for all } t \in[0, \widehat{t}[\text {. }
$$

In following, we state a comparison between $G_{0}, G_{1}$ and $G_{2}$ with the assumption of the equation (13) which indicates that in the following proposition where we clearly see the efficiency and the major interest given by the introduction of such functions.

Proposition 6. $G_{i}, i=0,1,2$, is strictly convex over $\left[0, \widehat{t}_{i}\left[, G_{i}(t) \rightarrow+\infty\right.\right.$ when $t \rightarrow \widehat{t}_{i}$. Beside, $-\infty \leqslant G_{2}(t) \leqslant G_{1}(t) \leqslant G_{0}(t) \leqslant \theta(t)$ for any $t>0$.

Proof. The first inequality is obvious. The inequality $\theta(t) \geqslant G_{0}(t)$ is proved in Theorem 4 .

Now, we prove that $G_{1}(t) \leqslant G_{0}(t)$. Let's consider the function $\nu(t)=G_{1}(t)-G_{0}(t)$. Since $\alpha_{0}=\tilde{\alpha}$ and $\alpha_{0} \leqslant \beta_{0}$, we have for any $t>0$

$$
v^{\prime \prime}(t)=\frac{\tilde{\delta} \tilde{\alpha}^{2}-(n-1) \alpha_{0}^{2}}{\left(1+\alpha_{0} t\right)^{2}}-\frac{\beta_{0}^{2}}{\left(1+\beta_{0} t\right)^{2}}=\frac{\beta_{0}^{2}}{\left(1+\beta_{0} t\right)^{2}}-\frac{\beta_{0}^{2}}{\left(1+\alpha_{0} t\right)^{2}} \leqslant 0 .
$$

Because $v^{\prime \prime}(t) \leqslant 0$ and $v^{\prime}(0)=0$, we have $v^{\prime}(t) \leqslant 0$ for all $t \in\left[0, \widehat{t}\left[\right.\right.$. Because $v^{\prime}(t) \leqslant 0$ and $v(0)=0$, we also have $v(t) \leqslant 0$ for all $t \in\left[0, \widehat{t}\left[\right.\right.$. Therefore, $G_{1}(t) \leqslant G_{0}(t)$ on $[0, \widehat{t}[$.

Next, we prove that $G_{2}(t) \leqslant G_{1}(t)$. Similarly, consider the function $w(t)=G_{2}(t)-G_{1}(t)$, so, $w(0)=w^{\prime}(0)=0$ and

$$
w^{\prime \prime}(t)=-\frac{\alpha_{2}^{2}}{\left(1+\alpha_{2} t\right)^{2}}+\frac{\tilde{\delta} \tilde{\alpha}^{2}}{(1+\tilde{\alpha} t)^{2}}=\|\lambda\|^{2}\left(\frac{1}{(1+\tilde{\alpha} t)^{2}}-\frac{1}{\left(1+\alpha_{2} t\right)^{2}}\right) \leqslant 0 .
$$

Because $w^{\prime \prime}(t) \leqslant 0$ and $w(0)=w^{\prime}(0)=0$, we have $w(t) \leqslant 0$ for all $t \in[0, \widehat{t}[$. Therefore $G_{2}(t) \leqslant G_{1}(t)$ on $t \in[0, \widehat{t}[$. The proof is complete.

Thus, we deduce that the function $G_{i}$ reaches its minimum at a unique point $\bar{t}_{i}$ which is the root of $G_{i}^{\prime}(t)=0$. for $i=1,2$, we have

$$
\bar{t}_{i}=\frac{\delta_{i}}{\gamma_{i}}-\frac{1}{\alpha_{i}} \quad \text { and } \quad G_{i}\left(\bar{t}_{i}\right)=\frac{\|\lambda\|^{2}}{\alpha_{i}}+\frac{\|\lambda\|^{2}}{\alpha_{i}^{2}} \ln (1-\beta) .
$$


In particular

$$
\bar{t}_{2}=\frac{-1}{\|\lambda\|-1} \quad \text { and } \quad G_{2}\left(\bar{t}_{2}\right)=\|\lambda\|-\ln (\|\lambda\|-1)^{-1} .
$$

The solution of the equation $G_{0}^{\prime}(t)=0$ returns us to solve the equation of the second order $t^{2}-2 b t+c t=0$, where

$$
b=\frac{1}{2}\left(\frac{n}{\gamma_{0}}-\frac{1}{\alpha_{0}}-\frac{1}{\beta_{0}}\right) \quad \text { and } \quad c=-\frac{\|\lambda\|^{2}}{\alpha_{0} \beta_{0} \gamma_{0}},
$$

whose roots are given by $t=b \pm \sqrt{b^{2}-c}$. For $\bar{t}_{0}$, we take the root that belongs to the interval $\left(0, \widehat{t}_{0}\right)$.

Thus, the three roots $\bar{t}_{0}, \bar{t}_{1}$ and $\bar{t}_{2}$ are explicitly calculated. Then we take $\bar{t}_{0}, \bar{t}_{1}$ and $\bar{t}_{2}$ are belongs to the interval $(0, \widehat{t}-\varepsilon)$, with $\varepsilon$ is a small positive real.

Lemma 3. Let $y_{k+1}$ and $y_{k}$ two strictly feasible solutions of perturbed problem (1), obtained respectively at the iteration $k+1$ and $k$, so we have

$$
f_{r}\left(y_{k+1}\right)<f_{r}\left(y_{k}\right)
$$

Proof. We have

$$
f_{r}\left(y_{k+1}\right) \simeq f_{r}\left(y_{k}\right)+\left\langle\nabla f_{r}\left(y_{k}\right), y_{k+1}-y_{k}\right\rangle
$$

and $y_{k+1}=y_{k}+t_{k} d_{k}$ thus

$$
f_{r}\left(y_{k+1}\right)-f_{r}\left(y_{k}\right) \simeq\left\langle\nabla f_{r}\left(y_{k}\right), t_{k} d_{k}\right\rangle=t_{k}\left\langle-\nabla^{2} f_{r}\left(y_{k}\right) d_{k}, d_{k}\right\rangle \simeq-t_{k}\left\langle\nabla^{2} f_{r}\left(y_{k}\right) d_{k}, d_{k}\right\rangle<0 .
$$

Therefore $f_{r}\left(y_{k+1}\right)<f_{r}\left(y_{k}\right)$.

\section{Conclusion}

In this study, we have presented a logarithmic barrier interior point method for solving the linear semidefinite programming problem. We have given the existence and uniqueness of the optimal solution of the corresponding perturbed problem and have verified its convergence to the optimal solution of the original problem when the barrier parameter approaches zero. Newton's method has been applied to find a new iterative point by calculating a sufficient descent direction. Due to the high computational cost, we have avoided using several methods, such as the line search methods, to calculate the displacement step. Alternatively, a new approach based on minorant functions has been proposed to accomplish this task to the optimal solution.

The minorant function technique is a very reliable alternative that will be confirmed as the technique of choice for both (SDP) and other classes of optimization problems.

The authors are very grateful and would like to thank the Editor-in-Chief and the anonymous referee for their suggestions and helpful comments which significantly improved the presentation of this paper.

\section{References}

[1] F.Alizadeh, J.P.Haberly, M.L.Overton, Primal-dual interior-point methods for semidefinite programming, convergence rates, stability and numerical results, SIAM Journal on Optimization 8(1998), 746-768.

[2] D.Benterki, Résolution des problèmes de programmation semidéfinie par des méthodes de réduction du potentiel, Thèse de doctorat, Département de mathématique, Université Ferhat Abbas, Sétif, 2004. 
[3] D.Benterki, J.P.Crouzeix, B.Merikhi, A numerical implementation of an interior point method for semide... nite programming, Pesquisa Operacional Journal, 23(2003), no. 1.

[4] S.Kettab, D.Benterki, A relaxed logarithmic barrier method for semidefinite programming, RAIRO-Operations Research 49(2015), no. 3.

[5] J.F.Bonnans, J.-C.Gilbert, C.Lemaréchal, C.Sagastizabal, Numerical optimization, theoritical and pratical aspects. Springer-Verlag, 2003.

[6] J.P.Crouzeix, B.Merikhi, A logarithm barrier method for semidefinite programming, R.A.I.R.O-Oper. Res., 42(2008), 123-139.

[7] J.Ji, F.A.Potra, R.Sheng, On the local convergence of a predictorcorrector method for semidefinite programming, SIAM Journal on Optimization, 10(1999), 195-210.

[8] T.Kim-Chuan, Some New Search Directions for Primal-Dual Interior Point Methods in Semidefinite Programming, SIAM Journal on Optimization, Aug., 2000.

[9] J.P.Crouzeix, A.Seeger, New bounds for the extreme values of a finite sample of real numbers, Journal of Mathematical Analysis and Applications, 197(1996), 411-426.

[10] Y.E.Nesterov, A.Nemirovski, Optimization over positive semidefinite matrices: Mathematical background and user's manual, Technical report, Central economic and mathematical institute, USSR academy of science, Moscow, USSR, 1990.

[11] R.T.Rockafellar, Convex analysis, Princeton University Press, New Jerzy, 1970.

[12] H.Wolkowicz, G.-P.-H. Styan, Bounds for eigenvalues using traces, Linear Algebra and Appl, 29(1980), 471-506.

\title{
Исследование логарифмического барьерного подхода для линейного полуопределенного программирования
}

Ассма Леуми

Кафедра математики Университет Скикда, Эдагария

Алжир

Башир Мерики

Джиамел Бентерки

Кафедра математики Университет Ферхата Аббаса Сефифа

Алжир

\begin{abstract}
В настоящей работе представлен логарифмический баръерный метод внутренней точки для решения задачи полуопределенного программирования. Метод Нъютона используется для вычисления направления спуска, а минорантная функция используется как эффективная альтернатива методам линейного поиска для определения смещения шага в направлении, чтобъ уменьшить порядок вычислений.
\end{abstract}

Ключевые слова: полуопределенное программирование, метод внутренней точки, метод логарифмического баръера, поиск строк. 